\title{
Artelogie
}

artelogie Recherche sur les arts, le patrimoine et la littérature de l'Amérique latine

$6 \mid 2014$

Horizons et dispositifs des arts plastiques des pays du Río de la Plata (XXe siècle)

\section{Le « voyage des Andes » des artistes du Río de la Plata au XX $\mathrm{XX}^{\mathrm{e}}$ siècle}

Jacques Poloni-Simard

\section{OpenEdition}

Journals

Édition électronique

URL : http://journals.openedition.org/artelogie/1294

DOI : 10.4000/artelogie.1294

ISSN : 2115-6395

Éditeur

Association ESCAL

Référence électronique

Jacques Poloni-Simard, « Le « voyage des Andes » des artistes du Río de la Plata au XXe siècle », Artelogie [En ligne], 6 | 2014, mis en ligne le 20 juillet 2017, consulté le 06 mai 2019. URL : http:// journals.openedition.org/artelogie/1294 ; DOI : 10.4000/artelogie.1294

Ce document a été généré automatiquement le 6 mai 2019.

Association ESCAL 


\title{
Le « voyage des Andes » des artistes du Río de la Plata au XX ${ }^{\mathrm{e}}$ siècle
}

\author{
Jacques Poloni-Simard
}

1 Le "voyage d'Europe » comme étape initiatique dans la formation et la carrière des artistes latino-américains est bien connu. Il s'effectuait grâce à des bourses, publiques ou privées, étanchait la soif de connaître les villes d'art européennes et leurs musées, se concrétisait par l'inscription dans les académies ou ateliers de peintres et la fréquentation des milieux artistiques des pays d'accueil. Si la destination française était privilégiée à la fin du xixe et au cours de la première moitié du xxe siècle, en raison de l'attraction de Paris comme capitale mondiale de la création et de l'innovation artistiques, l'Espagne, l'Italie, voire l'Allemagne ne doivent pas être oubliées dans cette géographie $^{1}$. Or il est une autre destination qui, certes secondairement, attira les artistes du Río de la Plata à partir des années 1920 : les provinces du Nord-Ouest argentin (Jujuy, Salta, Catamarca et Tucumán, regroupées sous l'appellation « NOA ») et les pays andins. Cette orientation nouvelle relève de la quête d'un "arte propio ", que celui-ci fût défini comme national ou américain ${ }^{2}$. Cette quête concerne aussi bien des artistes marqués par les débats du Centenario (opposant les tenants des traditions nationales aux défenseurs d'une ouverture internationale ${ }^{3}$ ) que ceux qui étaient soucieux de rendre compte d'une autre réalité sociale et anthropologique, ou qui cherchaient à promouvoir un nouvel art américain. Au-delà des positions intellectuelles et des postures esthétiques divergentes, leurs voyages et les centres d'intérêt que ceux-là manifestent sont à mettre en relation avec les débuts de l'anthropologie et les premières enquêtes sur les mythes et légendes des Indiens, l'art populaire, le folklore, avec ceux également de l'archéologie moderne, qui commence alors à étudier de grands sites préhispaniques : Tiahuanaco, Machu Picchu, pour certains d'entre eux (et non des moindres) nouvellement (re)découverts, ou à mettre en évidence l'existence de cultures régionales (par exemple, en Argentine, la culture diaguita).

2 Le choix de l'expérience des Andes, certes minoritaire et, selon les cas, alternative, première ou complémentaire du voyage d'Europe, fait advenir une autre Argentine, andine et non plus seulement gauchesque ou européenne, rattache le Río de la Plata au 
monde andin, met en relation les artistes uruguayens et argentins avec leurs collègues boliviens et péruviens, voire équatoriens ou colombiens, et en contact direct avec la préoccupation indigéniste de certains d'entre eux. À partir de quelques trajectoires d'artistes qui se tournèrent vers les hautes terres andines ou voyagèrent dans ces pays voisins, on verra comment étaient promus un autre paysage et une autre réalité humaine, qui tendait à constituer un nouvel horizon socio-culturel dans les débats sur l'identité de l'art argentin et uruguayen.

\section{L'art national, selon le modèle « Eurindia » de Ricardo Rojas}

Luis Perlotti (1890-1969) est un sculpteur qui s'est formé auprès de Lucio Correa Morales (1852-1923), qui avait été le premier à introduire des thèmes indianistes dans la sculpture argentine. À l'heure de sa formation artistique, Perlotti se tourna vers les cercles scientifiques qui commençaient à s'intéresser aux peuples indigènes, travaillant au ministère de l'Agriculture pour Eduardo Ladislao Holmberg (dessinant pour le bureau des Bosques y Yerbales), côtoyant le naturaliste Francisco Perito Moreno, le paléontologue Florentino Ameghino, et fréquentant le nouveau musée ethnographique qu'avait créé Juan Bautista Ambrosetti. Par la consultation de la littérature ethnographique et archéologique, et illustrant des ouvrages sur les tissus andins et la céramique préhispanique, Luis Perlotti se familiarisa avec les traditions culturelles indiennes et l'histoire des Amérindiens; il s'ouvrait ainsi à la thématique nativiste, à laquelle il resta fidèle tout au long de sa carrière. Dès 1918, au salon de la Sociedad Nacional de Artistas, Pintores y Escultores, Perlotti expose aux côtés du peintre péruvien indigéniste José Sabogal, alors à Buenos Aires, partage les cimaises avec les expressionnistes du groupe des Artistas del Pueblo, rencontre Benito Quinquela Martín, le "peintre de la Boca », avec qui il nouera une longue amitié. En 1923, il assiste à une représentation au Teatro Colón de la troupe de danses folkloriques cuzquéniennes ${ }^{4}$, dans le cadre de la Misión Peruana de Arte Incaico ${ }^{5}$, que dirigeait Luis Valcárcel ; celui-ci appartenait au cercle des indigénistes de Cuzco (aux côtés du photographe Martín Chambi et de l'écrivain José Uriel García, l'auteur de El Nuevo Indio - 1930).

4 Luis Perlotti voyagea beaucoup au cours de sa vie. Or il prit la route des Andes avant de se rendre en Europe. Dès 1924-1925, il est à La Paz, pour l'exposition internationale organisée dans cette ville, collaborant à l'envoi artistique de l'Argentine; et, depuis la Bolivie, il se rendit au Pérou. Il retourna dans les deux pays andins en 1935-1936, pour le 400e anniversaire de la fondation de Lima, visitant les principaux sites archéologiques de l'altiplano péruviano-bolivien, Tiahuanaco, Machu Picchu et la Vallée sacrée de Cuzco, mais aussi ceux de la côte (Nazca, Pachacamac). Dans son journal de voyage - aujourd'hui perdu -, il déclare, à propos de Cuzco : «Este viaje realizado a la ciudad de los recuerdos del pasado incaico, reafirma en mi alma mi profundo amor por la tradición de los Incas, que enriqueciría mi material ilustrativo pare el arte americanista ${ }^{6} . »$ Luis Perlotti revint encore au Pérou en 1962, poussant, depuis l'Équateur, jusqu'en Amérique centrale (Panama et Guatemala), effectuant d'autres voyages sud-américains au cours de sa vie : en Uruguay, en 1929; deux fois au Brésil - Rio de Janeiro -, où il exposa en 1936 et en $1937^{7}$. Ce n'est qu'à la fin de sa vie, en 1954, qu'il se rendit en Europe ${ }^{8}$ : en Italie, la terre de ses ancêtres, en Espagne et en France; dans les deux premiers pays cités, en 1967 à nouveau. Mais, plus encore que les pays andins, c'est toute l'Argentine que Perlotti a parcourue, de 
la province de Corrientes à celle de Patagonie, avec une prédilection pour les régions du Nord-Ouest. Au cours de ses voyages, il photographie, dessine, peint les paysages, les sites archéologiques et surtout les habitants de ces contrées, accumulant autant d'esquisses dont il s'inspirait lorsque, de retour dans son atelier, il sculptait les «types humains » de la population native sud-américaine.

Luis Perlotti fut un artiste reconnu en son temps. Il réalisa un nombre considérable de bustes des Pères de la Patrie, des hommes illustres de l'histoire argentine ou des grandes figures du monde des arts, des lettres et de la politique; il grava également de très nombreuses plaques et médailles commémoratives; il gagna aussi plusieurs concours pour la construction de monuments publics à forte connotation patriotique ${ }^{9}$, et pas moins de trente autres, dédiés à la Madre, ce qui l'amenait à se déplacer dans les cités argentines. La notoriété qu'il acquit de son vivant fut nationale; sa renommée fut aussi internationale ${ }^{10}$.

6 Luis Perlotti s'inscrit en fait dans le courant de pensée qui militait en ce début du xxe siècle pour l'affirmation d'un art national, et il adhérait aux idées de Ricardo Rojas, l'un des tenants, parmi les intellectuels de cette époque, du nationalisme dans tous les domaines artistiques. Celui-ci plaidait pour la fusion en une seule esthétique des formes héritées de la tradition classique européenne et de celles issues des traditions locales, exaltant le passé américain contre les modèles européens, d'où le néologisme qu'il forgea : «Eurindia ». Rojas résuma ses thèses dans l'un de ses ouvrages majeurs, au titre homonyme, et dont le sous-titre était : Ensayo de estética sobre las culturas americanas (1924) ${ }^{11}$.

7 Dans cet ouvrage, Ricardo Rojas précise ce qu'il entend par le concept d'Eurindia : « es el nombre de un mito creado por Europa y las Indias, pero que ya no es de las Indias ni de Europa, aunque está hecho de las dos"; "es el nombre de este nuevo misterio etnográfico, y la Argentina es, sin duda, el órgano más fecundo de tal creación »; " "Eurindia", que de ambas (l'Europe et les Indes) toma su nombre, intenta una refundición de las dos [tradiciones] $»^{12}$. Dans les débats qui agitaient les intellectuels autour de la question nationale, ébranlée par les bouleversements sociaux et culturels issus des vagues d'immigration qu'avait reçues l'Argentine à partir de la fin du xixe siècle, Ricardo Rojas se voulait le chantre d'une "argentinité » qui s'appuyât sur les traditions nationales, sans pour autant rejeter l'influence européenne ni se replier sur le seul substrat indigène. Dans les débats qui agitaient les milieux artistiques, il s'opposait tout à la fois au mimétisme académique à l'égard des modes européennes qu'au cosmopolitisme, que prônaient les avant-gardes modernistes : « En esa fusión reside el secreto de Eurindia. No rechaza lo europeo, lo asimila ; no reverencia lo americano : lo supera ${ }^{13}$. » Par ailleurs, le projet de Ricardo Rojas ne se limitait pas à la seule Argentine; son horizon était américain, et les perspectives qu'il traçait, il les déclinait aussi à cette échelle. Enfin, il y a chez Rojas une dimension américaniste, voire indigéniste, d'où son intérêt pour l'archéologie et l'anthropologie comme pour les arts préhispaniques et populaires. $\mathrm{R}$. Rojas reproduit ainsi la tension entre "civilisation et barbarie ", qu'avait théorisée Domingo Faustino Sarmiento en 1845, voyant quant à lui dans l'émancipation du monde des campagnes (Indiens, gauchos et caudillos, qui auraient préservé l'« esprit de la terre ») le socle de l'identité argentine, contre le monde des villes, attaché à l'Espagne et à sa culture étrangère. Si Rojas ne sous-estimait pas les bienfaits du nouvel «exotisme cosmopolite ", apportés par l'ouverture démographique de l'Argentine, il appelait de ses vœux et voyait poindre « los anuncios de una nueva creación autóctona, que no debe ser 
xenofobia marcial, sino creación pacífica de cultura americana, reivindicación nativista por medio de la inteligencia, conquista espiritual de nuestras ciudades por el genio americano ${ }^{14} »$. Cette affirmation nationale, nationaliste, partagée par beaucoup bien qu'exprimée en des termes différents, il la résumait ainsi : « No queremos ni la barbarie gaucha ni la barbarie cosmopólita. Queremos una cultura nacional como fuente de una civilización nacional»; et, pour ce qui est de l'art: «[queremos] un arte que sea la expresión de ambos fenómenos. "Eurindia" es el nombre de esta ambición »15, qu'il développait de la manière suivante: «Necesitamos, pues, una doctrina estética fundada en la experiencia de nuestra historia, que nazca aquí, para nosotros y para América, como afirmación de que la nacionalidad argentina ha llegado a sazón fecunda ${ }^{16}$.» Mais c'est l'affirmation de l'argentinité qui était la condition à ses yeux de la réalisation d'une nouvelle culture nationale et, au-delà, américaine : Eurindia, « con el Plata adelante, que abre el camino del mar hasta el horizonte del mundo; con la pampa detrás, que abre el camino de la tierra hasta el horizonte de América »; avec Buenos Aires pour capitale, à laquelle il manquait encore de «ligarse espiritualmente a nuestras provincias, esas catorce raíces que ella tiene adentradas en el continente para nutrirse en los profundos caudales de la Tradición Americana $»^{17}$.

8 Ricardo Rojas exprime ainsi un idéal américain, qui passe par une affirmation nationale : « América es la primera vía de nuestra expansión intelectual ; mas para llegar a ella se ha de partir de la propia nacionalidad ${ }^{18}$ ». Il diagnostique la nécessité d'une triple prise de conscience nationale et continentale, en raison de l'existence d'une communauté précolombienne (que l'archéologie mettait en lumière), d'une communauté coloniale (le sentiment d'un passé commun) et d'une communauté révolutionnaire (la lutte pour l'indépendance): "Somos americanos por la tierra, o argentinos, chilenos, peruanos, mejicanos, o lo demás que somos por la ciudadanía política »; " nos sentimos hispanoamericanos en razón de nuestra misma argentinidad »; « nuestro hispano-americanismo tiende a un panamericanismo $»^{19}$. Et il diagnostiquait en même temps qu'il annonçait l'aube de la prise de conscience d'une communauté esthétique, signe à ses yeux d'une future émancipation, spirituelle celle-ci. Dans le registre enflammé qui était le sien, Ricardo Rojas dénonçait tout à la fois la fascination aveugle à l'égard de tout ce qui était exotique et la dévalorisation de ce qui était autochtone ${ }^{20}$.

C'est à l'aune de ces conceptions qu'il faut lire l'œuvre sculptée de Luis Perlotti. À l'exaltation des traditions gauchesques (Martín Fierro, Santos Vega, Gaucho, El cantor) et nationales (El tango, Baile criollo, Pericón nacional), il associa celle des traditions andines (Danza de la flecha, Danza de los condores, Baile de la quebrada, Baile de la honda) et guaranies (Flor de Irupé). Car Perlotti développa une abondante œuvre indigéniste : il représenta des types sociaux (La tejedora, El chasqui, Changuito, Tejedoras) et indiens, auxquels il donnait le nom de groupes ethniques (Quechua, Aimara, Guaraní, Tehuelche, Calchaqui, Quilmes); promut le monde indien dans plusieurs de ses monuments publics : Los Andes (cf. ill. 1), El despertar de la raza, donnant même forme, dans ses plâtres, bronzes et pierres sculptées, à tout un univers préhispanique réinventé (Saludo al Sol, Inti, Oración). Si la facture de ses œuvres est toujours naturaliste, Perlotti y ajoutait des éléments décoratifs, en particulier géométriques, qu'il puisait dans l'analyse qu'il conduisait et l'observation qu'il avait faite, in situ, de l'art préhispanique ${ }^{21}$. 
III. 1 : Luis Perlotti, Los Andes, 1941

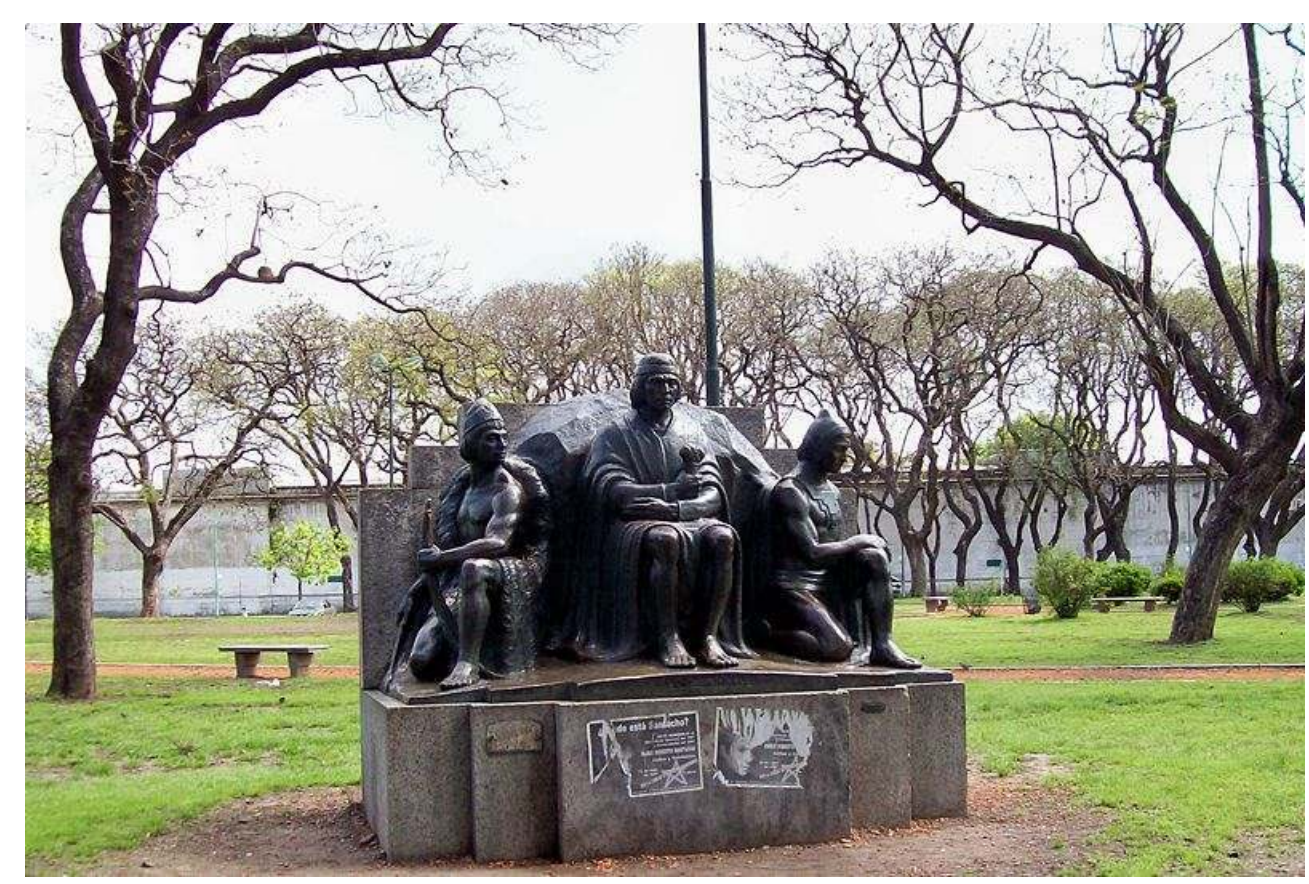

bronze, parc Los Andes (Chacarita, Buenos Aires)

Si, parmi tous les artistes - y compris les sculpteurs ${ }^{22}$ - qui exprimaient cette quête de lo propio, Ricardo Rojas ne donne pas Perlotti en exemple, il préfaça le catalogue de l'une de ses expositions, organisée en 1933 dans les locaux de l'association Ameghino à Luján ; Luis Perlotti y est qualifié, dès le titre, de « escultor de Eurindia " ${ }^{23}$. Rojas écrit : "Se ha dado en el la comprobación de que la tierra argentina es crisol de una raza y de una cultura nuevas. Lo que dilucidé en mi libro Eurindia, tórnase patente en casos como el suyo y en el de tantos otros artistas nativos, que han sabido refundir la tradición greco-latina de la civilización occidental en las ambiciones de un arte propio, con influencia de ambiente físico de América y de sus símbolos raciales ${ }^{24}$.» Parmi les artistes qui, selon Rojas, illustraient l'école « eurindienne $»^{25}$, Luis Perlotti avait toute sa place. Et, pour confirmer cette influence de Ricardo Rojas sur les milieux artistiques argentins, on aurait pu convoquer également le travail des architectes du courant néocolonial (Ángel Guido, Estanislao Pirovano, Martín Noel) ${ }^{26}$. Mais d'autres artistes, pendant ces mêmes années 1930 et 1940, ont posé un regard moins idéologique sur les Andes et leurs habitants.

\section{Regards socio-anthropologiques sur une autre Argentine}

11 À l'issue de son séjour en Europe entre 1925 et 1931, Antonio Berni (1905-1981), qui contribua à introduire le surréalisme en Argentine à la faveur de son exposition de 1932 à l'association Amigos del Arte, infléchit son œuvre dans le sens d'un réalisme social qu'il théorisa dans son manifeste "Nuevo Realismo », publié en 1936 dans la revue Forma, et nombre d'œuvres, parmi les plus célèbres de cet artiste ${ }^{27}$, illustrent sa préoccupation de peindre la réalité sociale argentine, avec une forte dimension critique. Il y déclare : « El Nuevo Realismo no es una simple retórica o una declamación sin fondo ni objectividad; 
por el contrario, es el espejo sugestivo de la gran realidad espiritual, social, política y económica de nuestro siglo ${ }^{28}$. " Cette ambition, Berni l'a affirmée comme relevant d'une responsabilité sociale, en tant qu'artiste argentin; ce programme, il l'a mis en œuvre par le choix de ses thèmes, à forte connotation socio-politique; cette orientation, il l'a considérée comme la seule réponse possible à la sempiternelle question, qu'il se posait aussi, de ce que devait être l'art argentin et, au-delà, latino-américain: «El Nuevo Realismo es nuestro y pertenece concretamente a América Latina ${ }^{29}$. Mais sans rien céder sur son appartenance aux courants de l'avant-garde portègne, Antonio Berni, rendant compte en 1976 de ce moment de sa trajectoire dans un long entretien avec José Viñals, y réaffirme son attention au contexte argentin pour expliquer sa prise de distance d'avec le surréalisme, si ses toiles en gardent la trace par les paysages métaphysiques dans lesquels sont inscrites les situations sociales qu'il dénonce ${ }^{30}$.

Or, dans cette perspective critique, Berni s'est aussi attaché à représenter d'autres Argentine que Buenos Aires et ses convulsions politiques. Si la province de Santiago del Estero est présente à son agenda pendant les années 1950, au cours desquelles il effectua plusieurs voyages et séjours, seul ou en compagnie de Juan Carlos Castagnino, Lino Enea Spilimbergo et Carlos Alonso, et dont il rapporta une série d'œuvres qui illustraient la condition socio-économique des paysans pauvres de la province ${ }^{31}$, Berni a aussi peint une toile où il évoque le Nord-Ouest andin de l'Argentine : Jujuy (1937, ill. 2), réalisée après son premier voyage dans cette région en 1936. Une seule œuvre ne suffit pas à convoquer ici son auteur, s'il n'y avait le séjour qu'Antonio Berni effectua dans les pays andins entre début janvier et fin mars 1942 (Bolivie, Pérou, Equateur, Colombie) ${ }^{32}$. Délégué de la Comisión Nacional de Cultura, il était chargé d'une étude sur l'art colonial hispanoaméricain et devait se rendre jusqu'au Mexique et aux États-Unis, la Sociedad Argentina de Artistas Plásticos (dont Berni présidait la commission pour la culture) lui ayant également confié la mission de rencontrer des artistes en vue de la tenue, à Buenos Aires, d'un congrès latino-américain d'arts plastiques (qui n'a pas vu le jour). Au cours de son voyage, Berni visita les sites archéologiques, s'intéressa, conformément à son ordre de mission, à l'art colonial (mais aussi à l'art populaire et préhispanique), prononça des conférences sur l'art moderne, la peinture argentine et américaine, insistant sur la dimension continentale de l'art à construire et auquel il souhaitait contribuer ${ }^{33}$. Il mettait tout particulièrement en avant sa collaboration avec David Alfaro Siqueiros pour l'exécution de la peinture murale Ejercicio Plástico (1933) dans la résidence de campagne du directeur du journal Crítica, Natalio Botana ${ }^{34}$. Dans les capitales qu'il visita, Berni réalisa des expositions et rencontra artistes et autorités locales en vue de favoriser les échanges artistiques, en particulier avec la Colombie. De ses quelque deux mois et demi passés dans les Andes, il rapporta une série d'articles, qui furent publiés dans La Prensa de Buenos Aires ${ }^{35}$, et, à partir de ses aquarelles réalisées sur place (vues de l'altiplano et scènes du quotidien), il peignit Mercado indígena (1942, aujourd'hui disparu). Cette œuvre, comme la précédente, témoigne de l'américanisme d'Antonio Berni et de son engagement pour un art sud-américain, qui reflète la réalité sociale, valorisant ici le monde andin par une thématique nativiste: "Hay que inspirarse siempre en la realidad. [...] Es indispensale que cada cuadro exprese una realidad social, y esto, no como militancia política, sino como militancia humana ${ }^{36}$.» 


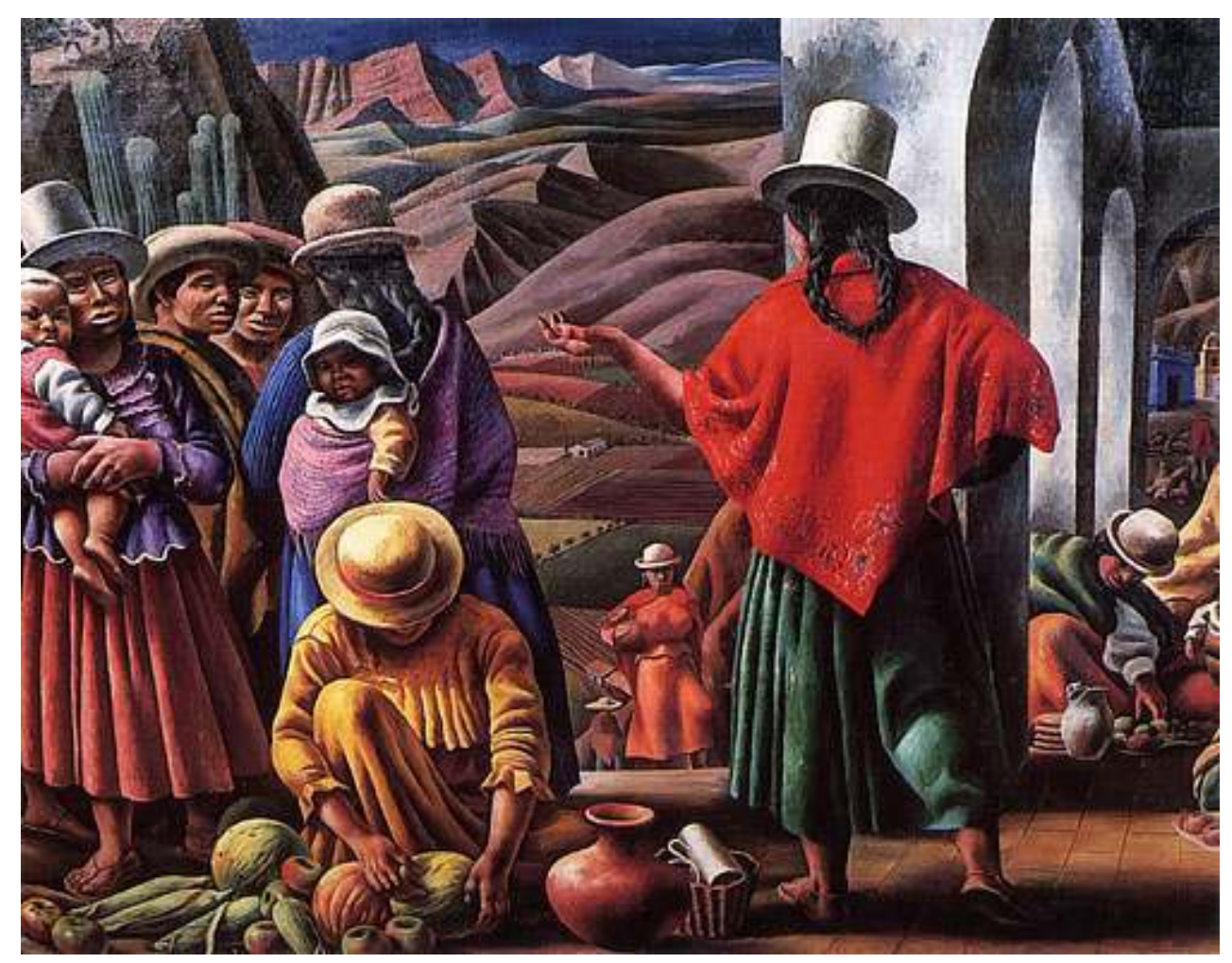

huile sur toile de jute, $190 \times 285$ cm, Museo de la Patagonia (Bariloche)

Après les Artistas del Pueblo (Guillermo Facio Hebequer, Adolfo Bellocq, Abraham Vigo, José Arato, Agustín Riganelli) qui ont gravé les ouvriers des usines et l'univers des basfonds et des miséreux de Buenos Aires, après Pío Collivadino et Benito Quinquela Martín qui ont fait de même en peignant les dockers du port de la Boca, Antonio Berni a contribué à faire entrer dans la peinture argentine d'autres réalités sociales, provinciales, à l'instar d'artistes comme Ramón Gómez Cornet (1898-1964, pour le Chaco), Adolfo Bellocq encore (1899-1972, pour la Patagonie et ses légendes), Enrique Policastro (1898-1971, pour la pampa et le Chaco, à nouveau). Participant lui-même des avant-gardes portègnes, Berni échappe à la tension nationalisme $v s$ cosmopolitisme ; sans pour autant entrer dans les débats sur l'art national, il relève cependant de la quête de lo propio, avec le fort accent critique qui le caractérise et son inquiétude quant à ce que doit présenter et représenter l'artiste argentin (puis latino-américain). Sans renier son expérience parisienne, Berni déclarait: «La pintura debe echar sus raíces en nuestra realidad actual", mettant en avant le souci d'inscrire son œuvre dans le contexte argentin « conviviendo con los hombres y con el paisaje argentinos " $^{37}$.

Il est une autre artiste, à l'itinéraire bien différent, dont la trajectoire argentine traduit une autre sensibilité, plus anthropologique celle-ci, mais dont les œuvres mettent également en valeur le monde andin, ses paysages et ses habitants. Il s'agit de Gertrudis Chale (1898-1954). Autrichienne, elle étudia à Vienne et Munich, travailla à Zürich comme décoratrice, à Paris dans les milieux de la publicité, à Madrid pour une maison de mode. C'est lors de son séjour aux Baléares qu'elle affirma sa vocation de peintre. À la suite de son mariage, elle effectua un premier séjour en Argentine en 1934, au cours duquel elle découvrit le Nord-Ouest, « [el] color maravilloso de estas tierras altas, diafanidad del aire, 
arquitectura de sus paisajes grandiosos, la gente que les animaba ${ }^{38}$ ». Revenue en Europe, qu'elle parcourut encore entre 1936 et 1938, sa judaïté la contraignit à revenir en Argentine, qui devint son pays d'adoption.

À Buenos Aires, Gertrudis Chale fréquenta les cercles artistiques de la colonie germanique, pour certains exilés comme elle : le dessinateur et graveur Clement Moreau (Carl Meffert), la photographe Grete Stern, le galeriste Rafí Sigman, le critique d'art Mauricio Neuman. Elle s'inséra aussi dans la communauté artistique et littéraire portègne, grâce à Oliverio Girondo et Norah Lange. En avril 1945, Gertrudis Chale débuta un voyage de dix-huit mois, qui la conduisit en Bolivie, au Pérou et jusqu'en Équateur. Dans les capitales de ces trois pays, elle exposa ses œuvres et celles de ses amis (Moreau et Stern, mais aussi Juan Carlos Castagnino et Horacio Coppola), établit des contacts avec les peintres indigénistes: les Péruviens José Sabogal, alors directeur du Museo de Arte Popular, et Julia Codesido, une élève de celui-ci; les Équatoriens Eduardo Kingman et Oswaldo Guayasamin, qui commençait sa période dite "Huacayñán». À son retour à Buenos Aires, G. Chale exposa les œuvres qu'elle avait peintes au cours de son voyage, ainsi que celles de ses nouveaux amis. Ce voyage andin la marqua profondément: elle chercha, sans succès, à publier le récit qu'elle en avait rapporté, se lança dans l'écriture d'un ouvrage sur les mouvements artistiques sud-américains et envisagea de fonder une revue, Vínculo, dont le sous-titre aurait été Revista indoamericana (de espaldas a Europa) ${ }^{39}$. Surtout, elle avait parcouru les Andes, visité les principaux sites archéologiques, séjourné dans des villages et participé à des fêtes (la Semaine sainte à Ayacucho). Elle écrira dans son journal de bord : «Viví con honda emoción inolvidables imágenes de la vida popular y mística del indio. Compartí sus fiestas en más de una ocasión ${ }^{40}$. » Au début des années 1950, Gertrudis Chale s'installa même dans la province de Salta, en compagnie des peintres Luis Preti, Raúl Brié et Héctor Julio Paride Bernabó (plus connu sous le nom de Vicente Carybé, lorsque celui-ci s'installa à Salvador de Bahia). Outre le Nord-Ouest, elle voyagea dans d'autres provinces argentines (le Chaco, la Patagonie) et en Amérique (Uruguay, Chili, Brésil, Mexique). Enfin, en 1954, peu avant sa mort dans un accident d'avion, elle participa à la décoration murale des Galeries Santa Fe, aux côtés de Raul Soldi, Juan Batlle Planas, Leopoldo Presas, Luis Seoane, Leopoldo Torres Agüero et Noemí Gerstein.

Gertrudis Chale est le peintre des Andes et des Indiens. Romualdo Brughetti a signalé à son propos «su deseo de investigar y de representar la realidad del hombre y del paisaje suramericano ${ }^{41} »$. Elle a parcouru l'altiplano et les vallées andines; elle s'est imprégnée de l'atmosphère des bourgs et des villages; elle y a surtout regardé leurs habitants. G. Chale insiste beaucoup sur le sentiment de "démesure " qui l'a saisie à la contemplation du paysage ( frente a cierto tipo de paisaje americano estamos en ambientes de “desmedida" $\left.{ }^{42} »\right)$. À la différence d'Antonio Berni, il n'y a pas de message social ou sociologique dans ses huiles et détrempes ; bien plutôt, il s'en dégage une forte sensibilité anthropologique (« antes de pintar cosas de ambiente americano trato de vivirlas y de impregarme de ellos $[$ sic $]$ » et «[...] aquí la vida y los hombres y su paisaje nos asaltan por todos los lados. Trato de captar una fracción de la realidad contemporánea $\left.»^{43}\right)$. À la différence de Luis Perlotti, G. Chale n'a pas dessiné les Indiens pour réaliser ensuite des «types humains", mais elle a cherché à les saisir dans leur quotidien («me importa llegar hasta su raíz más secreta y a su más esencial $\left.[\text { sic }]^{44} »\right)$. Il n'y a pas non plus chez elle de revendication indigéniste ou de dimension folklorique, mais une quête d'authenticité pour exprimer la réalité américaine, en l'occurrence andine: «Tiendo a que de mis 
cuadros trascienda un mensaje americano de nuestros tiempos y de nuestras latitudes ${ }^{45}$. " Ces paysages, subtilement colorés (cf. ill. 3), sont empreints d'une grande pureté (avec des réminiscences de la peinture métaphysique surréaliste), et ses personnages y sont posés ou les traversent, comme autant de représentants d'une humanité reléguée mais toujours noble, dans un environnement parfois qu'elle rapporte à Yves Tanguy. Elle traduit dans ses toiles son amour des Andes et manifeste une grande sensibilité à l'égard des réalités humaines, tout comme elle déclare sa flamme pour l'Indien (cf. ill. 4) ${ }^{46}$, sans pour autant succomber au folklorisme, comme le fait Alfredo Guido dans les fresques (peintes en 1925) du palais Fracassi, de Rosario, construit par son frère Ángel, et qui relèvent du projet « eurindien » de Ricardo Rojas.

III. 3 : Gertrudis Chale, Tormenta petrificada [Bolivie], 1945

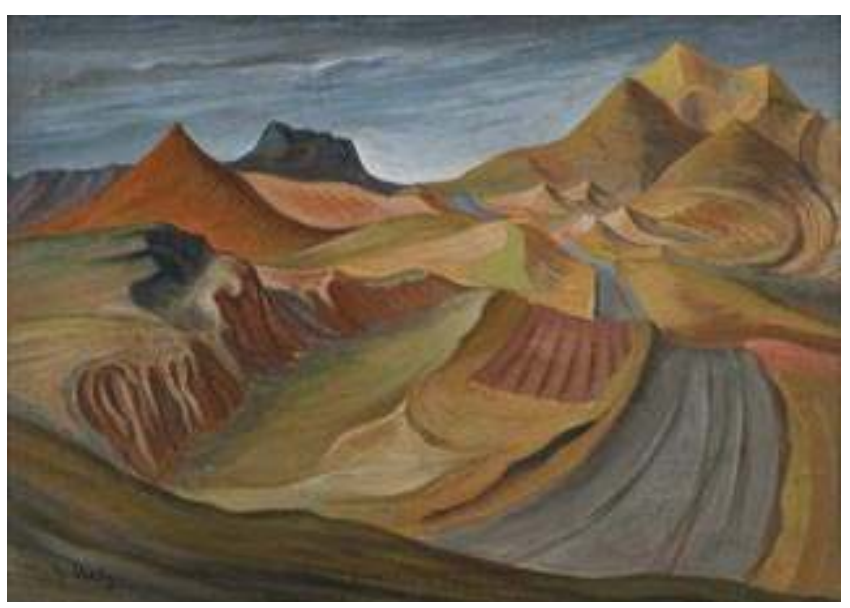

tempera sur bois, $50 \times 70,5 \mathrm{~cm}$

III. 4 : Gertrudis Chale, Dos figuras en el paisaje, 1943

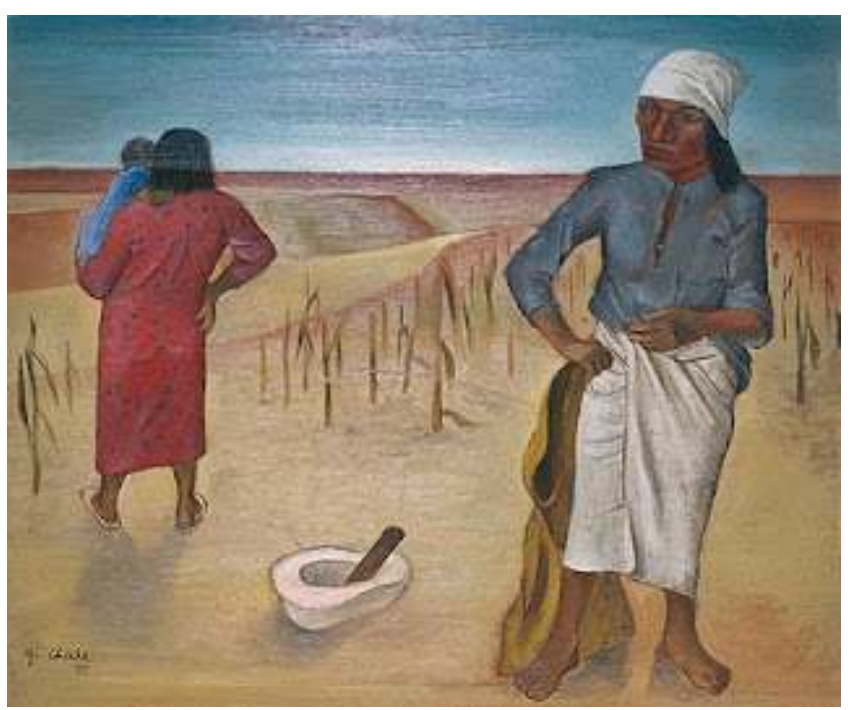

huile sur isorel, 43 × 50, coll. Francisco Traba (Buenos Aires)

Après avoir réalisé des paysages suburbains, c'est le paysage andin et la représentation de l'Indien qui ont retenu l'attention de Gertrudis Chale. Sur le paysage : «Pintando tal ambiente trato de insinuar algo de su tamaño físico: lo vasto, lo inmenso, lo insólito. En 
vez de llenar mis cuadros los vacio hasta dejar solo lo más significativo. Odio el "motivo" decorativo. Donde se encuentra el paisaje más depurado, la pampa y la puna, le hallo sus más sobresalientes cualidades estéticas ${ }^{47}$.» Et, sur l'Indien : "Por natural inclinación dediqué a observar la vida del indígena; me senté con él en las calles y mercados, haciendo miles de croquis para poder captar su modo de moverse, sus actitudes todas. Impresionada por la belleza de su figura, que hasta en su condición más miserable guarda extraordinario interés plástico, me he olvidado de su pobreza, de su condición social. He visto al indio incorporado a su tierra como parte de ella, llevando los colores de sus arenas, de sus cerros, guardada en sus ojos la desolación de la puna y en su alma la tristeza enorme de una raza quebrada en su destino humano ${ }^{48}$. »

Gertrudis Chale décrit ainsi ses voyages: «[...] viajes inmensamente interesantes, estimulantes por cuanto se veía, por el mismo contacto con la realidad del pueblo. Estimo que debería ser una experiencia que cada artista se propusiera deliberadamente y realizara a todo precio ${ }^{49}$. Dans les pages qu'elles nous a laissées, G. Chale critique le tropisme de ses collègues qui se tournent vers l'Europe ; elle partage les préoccupations des artistes soucieux de regarder les formes culturelles autochtones et indigènes; elle veut rendre compte par le pinceau de «lo americano", synonyme de "indio » sous sa plume. « Yo busco al paisaje y al hombre americano donde más se diferencia de los demás. Trato de reconocer sus elementos fundamentales y sus rasgos más característicos que demuestran que son americanos y SóLO americanos. Para lograr esto hay que conocer al no-americano ${ }^{50}$. » Gertrudis Chale nourrit ainsi une vision humaniste de l'Indien, qui l'a conduite à partager la vie de communautés, jusqu'à l'osmose, s'émouvant de la condition sociale des Indiens sans pour autant déboucher sur une critique sociale ou politique de celle-ci ; jusqu'à l'identification, établissant un parallèle entre l'histoire indienne et son histoire personnelle. Comme d'autres artistes du Río de la Plata (le sculpteur argentin Libero Badii [1916-2001], dont elle croisa la route à Arequipa, Lima, Tumbes et Quito ; le peintre uruguayen Norberto Berdía [1900-1984] qui, après sa période sociale et un long séjour au Mexique, se tourna vers les mondes indiens du Paraguay et des Andes; E. Policastro et R. Goméz Cornet - déjà mentionnés -, qu'elle connaissait), Gertrudis Chale se mit en quête d'une autre Argentine, vécue comme la rencontre d'une autre Amérique, partit à la recherche d'une altérité géographique et anthropologique, d'une autochtonie non européenne, dont elle mesurait la distance à l'aune de sa propre européanité. À propos de son installation à Salta, elle écrira qu'elle était partie en quête d'images qui rendent compte de "lo americano", signalant ainsi l'américanisation de cette artiste européenne.

\section{Conceptions constructives indo-américaines}

La même année qu'Antonio Berni, en 1942, trois jeunes artistes uruguayens, fils et élèves de Joaquín Torres García (1874-1949), peintre en Catalogne d'origine uruguayenne, puis artiste international par sa trajectoire qui le conduisit à New York, Paris - entre 1926 et 1932 - et Madrid, avant de revenir dans son pays natal en 1934, empruntaient également les routes de la Bolivie et du Pérou : il s'agit d'Augusto Torres (Tarrasa, 1913 - Barcelone, 1992), Horacio Torres (Livourne, 1924 - New York, 1976) et Alceu Ribeiro (Artigas, 1919 Palma de Mayorque, 2013). Quatre ans plus tard, c'est au tour de quatre autres membres du Taller Torres-García, qui prit la suite en 1943 de l'Asociación de Arte Constructivo, fondée en 1935 par le maitre uruguayen de l'art constructif, de prendre le chemin des 
Andes $^{51}$ : Julio Alpuy (Cerro Chato, 1919-2009), Gonzalo Fonseca (Montevideo, 1922 Seravezza, 1997), Jonio Montiel (1924-1986) et Sergio de Castro (Buenos Aires, 1922 Paris, 2012). L'Europe était en guerre, et quasiment tous ces peintres et sculpteurs qui viennent d'être mentionnés y voyagèrent dans les années 1950 ; mais après l'Espagne, l'Italie ou la France, certains poussèrent jusqu'en Grèce (A. Torres, Fonseca) et en Turquie (Fonseca), se rendirent aussi, au cours de cette même décennie, au Moyen-Orient (Alpuy, Fonseca), en Égypte (Alpuy, Fonseca, Francisco Matto [Montevideo, 1911-1995]), voire au Soudan (Fonseca). Qu'est-ce qui attirait ces artistes en Bolivie et au Pérou d'abord, puis vers ces terres lointaines et exotiques ? Pour eux, prendre la direction des Andes, pendant les années 1940, ne s'explique pas seulement par l'impossibilité conjoncturelle de connaître l'Europe et ses musées. Ces disciples de J. Torres García avaient des motivations particulières pour répondre ainsi à l'appel de l'altiplano ${ }^{52}$. Il s'agissait de voir de leurs propres yeux les trésors que Torres García leur avait enjoint, avec insistance et ferveur, de regarder, en particulier lorsqu'il développa son projet «Indoamérica " ${ }^{53}$, sans luimême les avoir vus in situ: les structures monumentales de l'architecture préhispanique, celles de Tiahuanaco, Cuzco et Machu Picchu; la géométrisation frontale des décors peints ou tissés des huacos et mantas; la symbolisation des figures représentées et leur signification mythologique; l'expression du sacré qui imprègne toutes les productions artistiques des anciens Péruviens et Boliviens, et dénote une société en harmonie avec les forces surnaturelles. Ces quatre idées, appliquées à l'art inca, ainsi que Torres concevait et lisait toutes les expressions artistiques primitives ou des périodes archaïques, renvoient à quelques-unes de ses principales conceptions esthétiques et philosophiques : le planisme, la structure, le symbolisme, la dimension spirituelle que la création artistique doit intégrer.

C'est en 1937/1938-1939 que Joaquín Torres García a développé son projet indoaméricain, repris en partie en 1941/1942-1944. Celui-ci réalise une ambition plus vaste et illustre son « Art constructif universel » (i. e. cosmique) ou « Universalisme constructif », que Torres García avait commencé à développer à Paris, à partir de 1929, entreprit de mettre en œuvre à Madrid en 1933, puis de diffuser à Montevideo à partir de $1934^{54}$, en formant à chaque fois un groupe d'art constructif, et qu'il prolongea jusqu'en 1948, alternant avec des phases qui semblent des retours à la figuration, qu'il a toujours cherché à intégrer dans son travail, où sa peinture se fait plus descriptive avec le port et la ville de Montevideo comme thèmes privilégiés ${ }^{55}$. Prenant appui sur une tradition, inca ou préinca, qu'il insère dans la Grande Tradition abstraite (tout à la fois géométrique, symbolique et métaphysique), dans laquelle Torres inscrit lui-même son ambition esthétique $^{56}$, le moment indo-américain est en fait une mise en œuvre située de son art constructif universel ${ }^{57}$. S'il s'est attaché, à Madrid comme après son installation en Uruguay, à l'étude des sociétés préhispaniques ${ }^{58}$, ce n'est que dans un deuxième temps qu'il a fait de la tradition andine, la seule à ses yeux originale en Amérique (c'est-à-dire hors de toute influence de la tradition européenne), le point d'appui de son enseignement, afin d'ancrer, en et pour l'Amérique ${ }^{59}$, son utopie constructive universelle. Il trouvait, dans les Andes, bien plus qu'un répertoire de formes à intégrer, une tradition à revendiquer ou une identité à affirmer : un nouvel horizon mythologique ${ }^{60}$, à même d'enrichir sa propre cosmogonie, de renouveler son projet intégral (pour être mieux compris), et de donner un nouvel élan à son ambition, sur des bases américaines. Il renouait ainsi avec certaines de ses postures lorsque, bien des années auparavant, à 
Barcelone entre 1911 et 1916, il s'était donné la Grèce comme horizon mythologique pour fonder son moderne classicisme méditerranéen ${ }^{61}$.

21 L'injonction à se mettre à l'école des époques inca et préinca trouve place dans le projet utopique de Joaquín Torres García, qui était rien moins que de refonder toute l'histoire de l'art, en retournant aux sources de la création plastique, d'embrasser et dépasser les avant-gardes du xxe siècle, parce que, comme l'exprime Juan Fló, « el arte va más allá que el arte -o retorna a ese más allá6 ${ }^{62}$. Et c'est ainsi que, ancrée dans une tradition représentative de la Grande Tradition abstraite, le nouvel art (constructif et moderne) pourra fleurir depuis l'Uruguay, s'épanouir dans toute l'Amérique et rayonner au-delà, pour garder la métaphore solaire, chère à Torres. Car, du point de vue d'une géographie artistique mondiale, l'épicentre de l'innovation basculait avec lui vers un «nouveau Nouveau Monde ", l'heure du continent américain semblant sonner, au moment où la culture européenne s'abîmait dans la guerre ${ }^{63}$.

Joaquín Torres García a ainsi contribué à la redécouverte des arts préhispaniques, s'il le fit dans une perspective bien différente de celle des indigénistes péruviens et mexicains, et à l'intérieur d'un système de pensée qui lui est propre. Son œuvre, à son tour, permet de mettre en évidence les sources préhispaniques de l'art moderne en Amérique, du Sud comme du Nord ${ }^{64}$, si, pour lui, les feux qui sont braqués sur son moment indo-américain ont fini par occulter le fait que celui-ci ne constitue qu'une phase de son art constructif et une variante de son universalisme constructif. Si l'on se tourne d'ailleurs vers l'une des œuvres emblématiques du grand projet esthétique torrésien, et qui a valeur de manifeste, le Monumento Cósmico - (1935) 1936 [1937] ${ }^{65}$ - installé dans le parc Rodó de Montevideo, on doit se rendre à l'évidence qu'il n'illustre pas l'américanisme de Joaquín Torres García : cette paroi de blocs gravés ne porte pas de symboles indo-américains; ceux-ci apparaitront plus tard dans son répertoire de signes : les masques de Pachamama/Tierra et de Viracocha, la pyramide à degrés, le lama. - Les élèves qui ont suivi Joaquín Torres García dans la mission que celui-ci s'était fixé de promouvoir en Uruguay un nouvel art tout à la fois primitiviste et moderne, et de le proposer, depuis Montevideo, à toute l'Amérique, ont accompagné le tournant indo-américain du maître, mais sans perdre de vue l'ambition qui le surplombe. Et c'est bien cette leçon de son enseignement et de son exemple qu'ils ont retenue quand ils ont développé leur œuvre personnelle, le projet «Indoamérica » étant comme enchâssé dans l'universalisme constructif plus vaste de Torres García ${ }^{66}$.

23 À l'exception notable de F. Matto, presque tous les disciples de la première heure furent mobilisés en 1944 pour participer à la décoration du pavillon Martirené, nouvelle unité destinée aux patients atteints de tuberculose, de l'hôpital Saint Bois: un ensemble composé de trente-cinq peintures murales (murales) ${ }^{67}$. Torres en réalisa lui-même sept ${ }^{68}$, faisant référence à la mythologie andine dans l'une d'entre elles: Pacha Mama (ill. 5). CEuvre emblématique, à l'instar du Monumento cósmico, mais en ce sens, cette fois, qu'il s'agit d'une réalisation collective (sinon anonyme ${ }^{69}$ ), comme Torres García le souhaitait lorsqu'il parlait du groupe d'art constructif, publique, remplissant ainsi la fonction sociale qu'il avait de l'art, et murale, ce qu'il avait toujours mis à son agenda artistique, depuis ses années barcelonaises et les fresques du salon Sant Jordi du palais de la Généralité de Catalogne, exécutées entre 1913 et $1916^{70}$. Les artistes constructifs qui voyagèrent dans les Andes en 1942 et 1945 réalisèrent les œuvres suivantes : Augusto Torres : Almuerzo (190 x $205 \mathrm{~cm})$ et Ferrocarril $(190 \times 490 \mathrm{~cm})$; Horacio Torres: Puerto, ou El barco $(180 \times 490 \mathrm{~cm})$, et Hombre, ou Composición con motivos indoamericanos (190 x $190 \mathrm{~cm})$; Alceu Ribeiro : Ciudad 
$(100 \times 260 \mathrm{~cm})$; Julio Alpuy : Ciudad $(200 \times 300 \mathrm{~cm})$ et Composición $(108 \times 266 \mathrm{~cm})$; Gonzalo Fonseca : Café $(130 \times 190 \mathrm{~cm})$ et Ciudad $(190 \times 375 \mathrm{~cm})$; Sergio de Castro : Casa (140 x 185 $\mathrm{cm})$ et Mar (190 x $275 \mathrm{~cm})$; Jonio Montiel n'en réalisa aucune. Peintures constructives plus qu'œuvres d'art constructif universel à proprement parler ${ }^{71}$, l'ensemble est d'une grande cohérence par la palette utilisée (où dominent les trois couleurs primaires, plus le noir et le blanc) et par la thématique (la ville, le port, les objets du quotidien ou les moyens de transport). - Joaquín Torres García associe d'ailleurs les deux réalisations, les murales de l'hôpital Saint Bois et le Monument cosmique, et les revendiqua comme des exemples aboutis de son utopie artistique: "Sí, lo que busqué siempre, fué el arte monumental decorativo $^{72}$, con sentido general humano, religiosamente laico, colectivo y lindando con el artesanado ; arte de pueblo y no de clase. Y yo me felicito de que haya sido aquí, en mi patria, que yo haya podido realizar plenamente tan profunda aspiración: son el Monumento Cósmico de [sic] Parque Rodó, y los grandes Murales del Pabellón Martirené en la Colonia Saint Bois ${ }^{73}$.».

\section{5 : Joaquín Torres García, Pacha Mama, 1944}

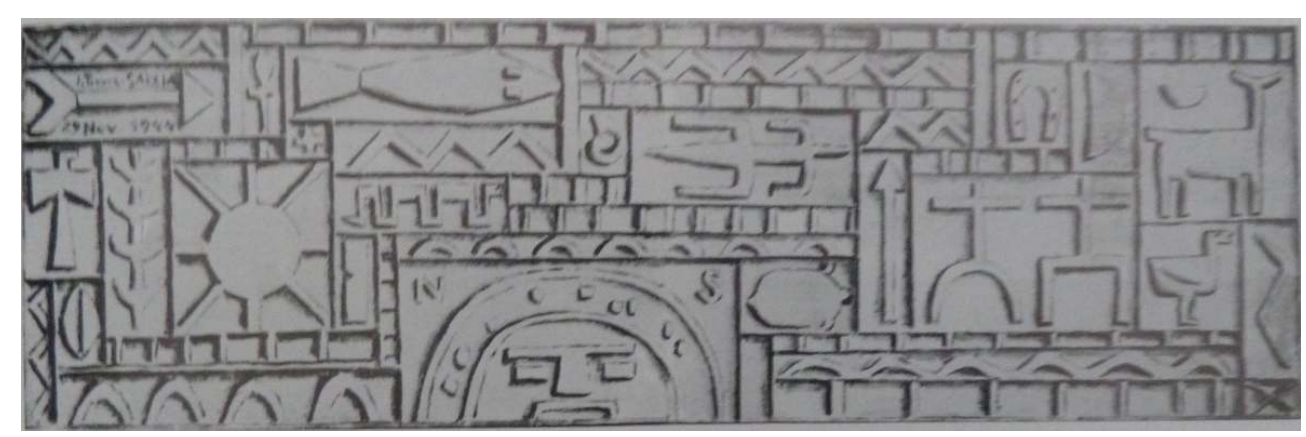

fresque du pavillon Martirené de l'hôpital Saint Bois

(voir Torres-García. Obras destruidas en el incendio del Museo de Arte Moderno de Rio de Janeiro, Mtv, Fundación Torres-García, s. d. [1981], ill. 4, pp. 28-29 et 105)

C'est dans le cadre du Taller Torres-García que travaillèrent les artistes voyageurs. Celuici prit la suite en 1943 de la AAC, après que Torres, déçu de n'avoir pu convertir à ses conceptions philosophiques et esthétiques le milieu artistique uruguayen, eut dissous l'Association en 1939 et décidé de se retirer dans son atelier ${ }^{74}$. Le Taller poursuivit ses activités jusqu'en 1963. Ses membres, conformément à l'enseignement du maître, approfondirent l'étude de l'art inca, organisèrent nombre d'expositions collectives auxquelles participait Torres García, réalisèrent des travaux d'arts décoratifs et appliqués ${ }^{75}$, firent des incursions dans l'architecture et continuèrent l'ambition d'un « art pour tous », mural. Le groupe publia également une revue, Removedor (28 numéros parus entre 1945 et 1953, Guido Castillo étant le responsable éditorial), où ils défendaient de manière virulente l'art constructif torrésien, en tant que mouvement artistique d'avant-garde en Amérique. - Parmi les artistes constructifs qui voyagèrent dans les Andes en 1942 et 1945 , on peut en signaler quelques-uns, dont certaines œuvres témoignent, pendant les vingt années au cours desquelles fonctionna le TTG, de leur adhésion au projet indoaméricain. Pour autant, il ne faut pas les réduire à cette seule dimension. D'une part, ils accompagnèrent l'évolution de leur maitre, inscrivant davantage leurs productions dans la phase de peinture constructive que dans celle de l'art constructif universel de Torres García, et, pour ceux qui poursuivirent une carrière artistique, développèrent leur style propre, tout en gardant la marque, plus ou moins forte, de l'enseignement torrésien. 
Le fils cadet de Torres García, Horacio Torres s'est engagé dans le projet indo-américain de son père: en 1939, il sculpte Ídolo en granito rosa, dans le style des figurines de la culture Valdivia (côte occidentale de l'Équateur, 3500 - 1800 av. J.-C.). Plus tard, en 1962, il assura la décoration intérieure de l'église du séminaire archidiocésain de Toledo, construit en briques par Mario Payssé Reyes. Le mur plat de l'abside, de briques également, est dans le style des "Constructifs» (Constructivos) tubulaires de son père: trame de lignes orthogonales définissant des rectangles de surfaces irrégulières, dont les bords latéraux sont arrondis. L'artiste a également représenté deux symboles du répertoire constructif torrésien, légèrement incrustés à l'entrée de la nef : Pez et Triángulo (ou Trinidad). Julio Alpuy et Augusto Torres continuèrent le rêve de Joaquín Torres García d'un art public: le premier peignit, par exemple, un mural dans une bâtiment de l'hippodrome de Maroñas (1951); le second en réalisa un autre dans la cafétéria du syndicat des médecins de Montevideo (1954). Comme Torres García, Gonzalo Fonseca dessina une carte de l'Amérique du Sud: Mapa de América del Sur (1950), orientée conformément au nord magnétique indiqué par la boussole, elle-même représentée sur le dessin. Sur le tracé du continent sud-américain, quadrillé par les parallèles et les méridiens, sont figurées et désignées les principales cultures préhispaniques. À hauteur du Río de la Plata se trouve l'indication "T.T.G.", et son emblème, le poisson. Les sculptures « archéologiques » de cet artiste, où l'on peut retrouver l'influence de ce qu'il a vu dans les Andes, au Moyen-Orient et dans la vallée du Nil, sont plus tardives : elles datent des années 1970. C'est sans doute Francisco Matto, peintre - marqué par Paul Gauguin avant sa rencontre avec Torres García - et sculpteur, qui est resté le plus proche de l'art constructif indo-américain ${ }^{76}$, avec les Totems qu'il réalisa entre 1970-1988. Évoquant les rewe mapuche, qu'il avait vus dans le sud du Chili, ses bois peints reprennent ou inventent des symboles constructifs : $U$, Cordero, Rostro humano, Tablas de la ley, Venus, Venus azúl, Caracol, Forma, pour n'en citer que quelques-uns (cf. ill. 6). Quant à Sergio de Castro, un an avant sa mort à Paris en 2012, il évoquait encore la mémoire de Julio Alpuy, Gonzalo Fonseca et Jonio Montiel, et ravivait dans un dessin le souvenir de leur voyage andin (cf. ill. 7). 
III. 6 : Francisco Matto, Totems (U, Venus azul, Rostro humano, Caracol, Venus)

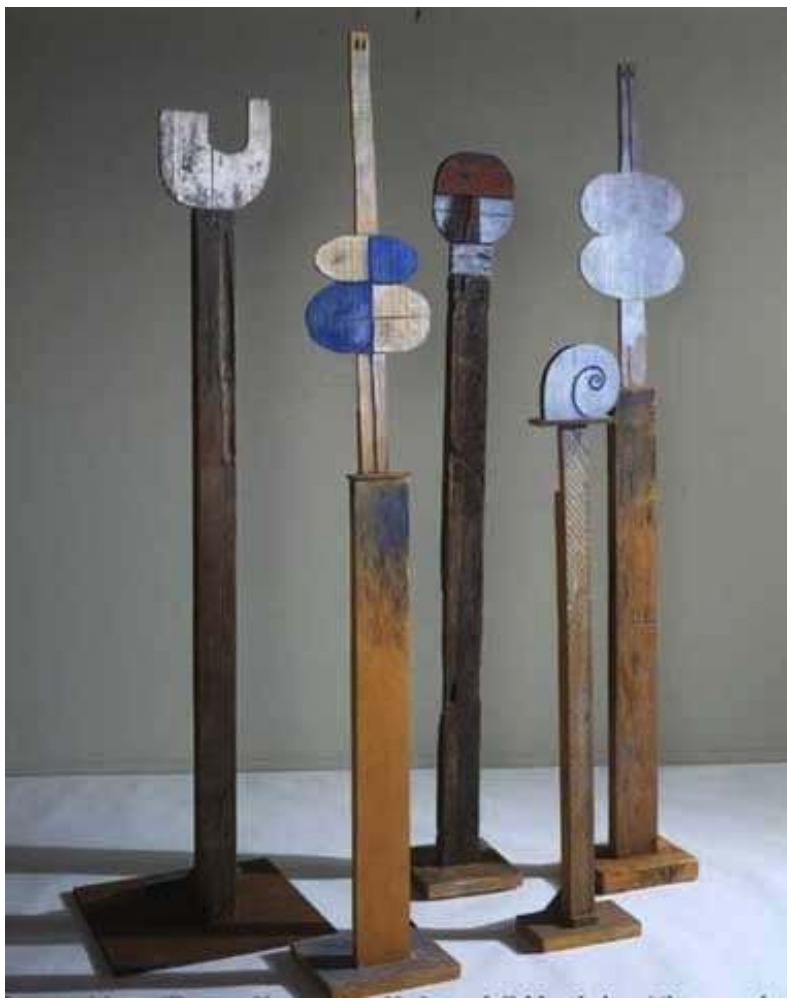

bois peints, diff. dates et tailles

III. 7 : Sergio de Castro, Inscription. Le « Voyage précolombien », 2011

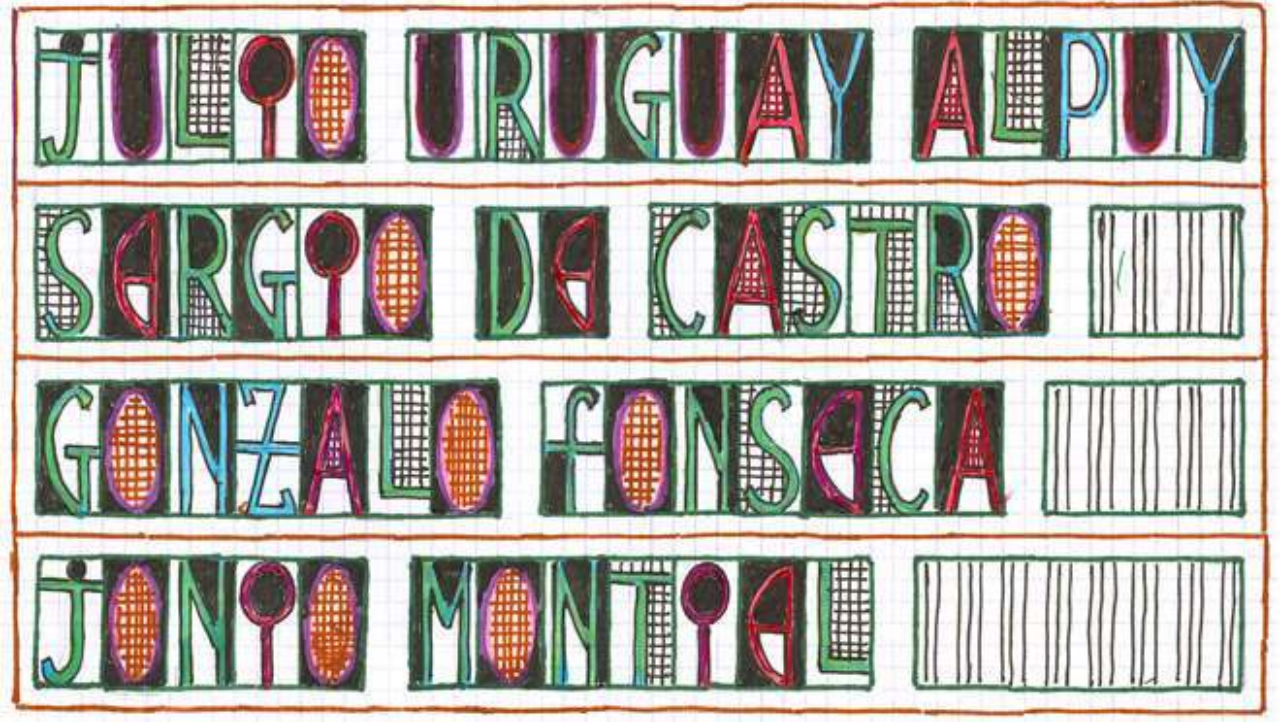

TAStNo 2011

11.265

encre sur papier, 29,7 × $21 \mathrm{~cm}$, coll. partic. (Paris) 


\section{Conclusion}

Ainsi, à l'instar du "paradigme amérindien » comme l'une des sources de l'abstraction américaine ${ }^{77}$, on peut parler d'un " paradigme andin ", pour un certain nombre d'artistes argentins et uruguayens des années 1920, 1930 et 1940, aussi différents que Luis Perlotti (ou Alfredo Guido), Antonio Berni, Gertrudis Chale ou Joaquín Torres García et ses disciples. S'il s'est exprimé dans des styles opposés, qui vont du naturalisme jusqu'au constructivisme, la référence préhispanique, andine, mais aussi native, indienne, est prégnante, qu'elle se manifeste par le recours à un vocabulaire décoratif géométrique, le choix d'une thématique socio-anthropologique ou l'expression d'une conception primitiviste de l'art moderne. L'appel à retrouver la tradition artistique andine et préhispanique n'a certes pas le même sens pour Ricardo Rojas, Antonio Berni ou Torres García, et sa valorisation s'inscrit dans des projets esthétiques et culturels bien différents, qu'ils relèvent de la quête d'un art national, de l'impératif de représenter la réalité sociale nationale, dans toute sa diversité, ou de la promotion d'un nouvel art d'Amérique. Pour autant, cette référence andine et ce «voyage des Andes» signalent une ligne de force présente chez nombre d'artistes argentins et uruguayens, qui relie le Río de la Plata aux Andes et articule les propositions eurindienne, néo-réaliste et indo-américaine aux indigénismes péruvien, bolivien et, au-delà, mexicain.

\section{NOTES}

1. Voir, par exemple, Territorios de diálogo. México, España, Argentina 1930-1945 (Entre los realismos y lo surreal), Buenos Aires [désormais BA], Fundación Mundo Nuevo, 2006 (cat. exp.).

2. Cf. Ivone Pini, En busca de lo propio. Inicios de la modernidad en el arte de Cuba, México, Uruguay y Colombia 1920-1930, Bogotá, Universidad Nacional de Colombia, 2000.

3. Voir Óscar Terán, «El modernismo cultural (Manuel Gálvez y Leopoldo Lugones) y El juicio del siglo de Joaquín V. González », in Id., Historia de las ideas en la Argentina. Diez lecciones iniciales, 1810-1990, BA, Siglo Veintiuno Editores, 2008, leçon 6, pp. 155-189; Carlos Altamirano (dir.), Historia de los intelectuales en América Latina, vol. I, La ciudad letrada, de la conquista al modernismo, BA, Katz Editores, 2008.

4. Luis Perlotti fit le portrait de l'une des danseuses, La niña del Cuzco (1924), et réalisa ses premières sculptures relevant d'une thématique indienne : Sulkapuma (1924), Inti (1932).

5. Cf. Elizabeth Kuon Arce et alii, Cuzco - Buenos Aires. Ruta de intelectualidad americana (1900-1950), Lima, Universidad de San Martín de Porres, 2009, pp. 205-216.

6. Cité dans Sara Saenz Cavia de Morales Torres, Luis Perlotti, el escultor de América. Biografía novelada, BA, Nelson Editorial, 1971, p. 160.

7. Depuis les studios d'une radio carioca, Luis Perlotti donna une conférence sur le thème : «Los artistas de Perú y Bolivia y la influencia de los artistas contemporáneos sobre el arte del pasado americano » (signalée dans Ibid., pp. 170-171).

8. En 1937, il avait participé à l'exposition itinérante (Rome, Milan, Madrid, Paris) : « Exposición Indigenista Americana del escultor argentino Luis Perlotti, España-Italia-Francia ». 
9. Il participa en 1925, avec Héctor Greslebin, architecte néo-préhispanique, au concours lancé pour la construction d'un monument à l'Indépendance à Humahuaca, remporté finalement par Ernesto Soto Avendaño. Sur ce courant architectural, voir Daniel Schávelzon, «Una corriente artística desaparecida : Héctor Greslebin y el arte neoprehispánico argentino (1915-1935) », 1986,

10. Outre Montevideo et Rio de Janeiro, Perlotti exposa à Valparaiso, Asunción, La Paz, Lima, où certaines de ses œuvres sont conservées, tout comme au Costa Rica, au Guatemala, au Mexique et à Cuba, et jusqu'aux États-Unis, en Italie et en Grèce ; sans doute y en a-t-il également en Espagne et en France.

11. Voir aussi Ricardo Rojas, Silabario de la decoración americana, BA, Librería 'La Facultad', J. Roldán y Cia., 1930.

12. Id., Eurindia. Ensayo de estética sobre las culturas americanas, BA, Editorial Losada, [1924] 1951, pp. 12 et 81 .

13. Ibid., p. 128.

14. Ibid., p. 21.

15. Ibid., p. 21.

16. Ibid., pp. 37-38, et : « No temamos volver a nuestra tierra [...]. Por nuestro arte iremos al de América, y por América a la Humanidad » (p. 38).

17. Ibid., p. 150.

18. Ibid., p. 69.

19. Ibid., pp. 61 et 66 .

20. «Los dioses de América se manifestaron en los tiempos de los Incas ; pero se alejaron de la tierra amedrentados por los crímenes del conquistador militar y por los exorcismos del evangelio católico. Volvieron otra vez a manifestarse en tiempo de los Libertadores, y se alejaron más tarde, aquí en la Argentina, corridos por el sensualismo del colonizador industrial y por la hostilidad del sabio materialista. Veo, sin embargo, claros indicios de que los dioses de América rondan otra vez muy cerca de nosotros, sugiriendo nuevas formas estéticas [...]. Uno de esos dioses americanos es aquel espíritu o fuerza que llamamos "Argentinidad" » (Ibid., pp. 121-122).

21. Perlotti suivait ainsi l'inventaire qu'en avait fait Ricardo Rojas dans Silabario de la decoración americana, op. cit.

22. Le précurseur du nationalisme en sculpture est à ses yeux Correa Morales, auquel il associe ses deux principaux disciples : Rogelio Yrurtia (1879-1950) et Pedro Zonza Briano (1866-1941).

23. Cette présentation est reprise dans Ricardo Rojas, Luis Perlotti escultor de Eurindia, BA, s. éd., 1935 (rééd. BA, Collana Artística, 1962).

24. Ibid., pp. 6-7.

25. "Mas el núcleo glorioso de la actual escuela "euríndica", lo constituyen Bermúdez, Quirós y Fader, por la índole de sus temas y por la conciencia doctrinaria de su misión estética en la patria. Con iguales propósitos, trabajan Octavio Pinto, Alfredo Guido, Antonio Alice, Rodolfo Franco, Gramajo Gutiérrez, Quinquela Martín y muchos más que ya forman phalange, [...] escuela que va caracterizándose por su valor artístico y por su función histórica en la cultura argentina » (Id., Eurindia..., op. cit., pp. 232-233).

26. Voir, par exemple, Martín Noel, Fundamentos para una estética nacional. Contribución a la Historia de la Arquitectura Hispano-Americana, BA, Talleres de Rodríguez Gilles, 1926, et El arquitecto Martín Noel : su tiempo y su obra, Séville, Consejería de Cultura, 1995 (cat. exp.).

27. Manifestación (1934), Desocupados (1934), Chacareros (1935/36).

28. Antonio Berni, «El Nuevo Realismo », Forma. Revista de la Sociedad Argentina de Artistas Plásticos (BA), 1, août 1936, reproduit dans Berni. Escritos y papeles privados, BA, Temas Grupo Editorial, 1999, pp. 79-82, ici p. 82.

29. Berni. Entrevista con José Viñals, BA, Imagen Galería de Arte, 1976, cité dans Berni. Escritos..., op. cit., p. 76. 
30. «Llego acá y me enfrento con dos realidades: en el orden intelectual, un colonialismo indiscutible que continúa todavía ; en el orden sociopolítico y económico, una situación de crisis profundísima », et, plus loin, « el surrealismo, como expresión estética pura, ya no tenía lugar en mí ; yo tenía la sensación de que no decía nada y que no me servía para nada » (Ibid., pp. 78 et 79). 31. Los hacheros (1953), La marcha de los cosecheros (1953), Escuelita rural (1956), La familia (1957), La comida (1953), Migración [ou Los Migrantes] (1954), Los algodoneros (1956). Louis Aragon, avec lequel Berni avait noué amitié, fit l'éloge de cette série, lorsque certaines de ces toiles furent exposées à la galerie Creuze de Paris en 1955 : voir «El quebracho... », Bulletin de la Galerie Raymond Creuze, octobre 1954 à février 1955, pp. 45-48.

32. Sur ce voyage, voir Cecilia Rabossi, «Antonio Berni : los periplos hacia la realidad », in Berni y sus contemporáneos. Correlatos, BA, Malba - Colección Costantini, 2005 (cat. exp.), pp. 33-38 ; María Florencia Galesio et Paola Melgarejo, « Entre utopías y realidades. El viaje de Antonio Berni por la América andina y su presencia en el MNBA », in Roberto Amigo (éd.), Berni : narrativas argentinas, BA, MNBA, 2010 (cat. exp.), pp. 191-205.

33. " He elegido temas americanos hallados durante mis recorridos, especialmente sobre base de inspiración peruana y boliviana que tiene una notable afinidad con nuestro ambiente argentino » ( El Nuevo Realismo en pintura orientará el arte americano. Antonio Berni nos habla sobre su obra de arte », La Razón [Bogotá], 23 mars 1942, entretien avec Antonio Berni : Fundación Espigas, Buenos Aires, Archivos Especiales, Antonio Berni, Carpeta Berni 2, 1941-1943, p. 169).

34. Voir Jacques Poloni-Simard, «Le muralisme des années 1930 et 1940 dans les pays du Río de la Plata », Nuevo Mundo - Mundos Nuevos, 14, 2014 <http://nuevomundo.revues.org/66328>.

35. «El pintor colonial Melchor Pérez de Holguín", La Prensa (BA), 15 février 1942 (in Berni. Escritos..., op. cit., pp. 187-188) ; «El arte de la época colonial. Gorivar, un pintor quiteño », La Prensa (BA), 31 mai 1942 (in Ibid., pp. 183-184) ; «Cuatro pintores modernos peruanos », La Prensa (BA), 27 septembre 1942 (in Ibid., pp. 190-191) ; «La escultura quiteña de la Época Colonial », La Prensa (BA), 25 avril 1943 (in Ibid., pp. 184-187).

36. «Antonio Berni y su pintura de realismo trascendente ", El Bien público (Mtv), 14 septembre 1938 (Fundación Espigas, Archivo Berni, Carp. 1, 1920-1940, p. 103).

37. "Antonio Berni busca en la realidad el tema y la emoción para su obra de artista », Desfile (BA), 7 novembre 1941 (Fundación Espigas, Archivo Berni, Carp. 2, 1941-1943, p. 163).

38. Citée par Julianne Gilland, "Gertrudis Chale. Perspectiva sobre su vida y su obra ", in Mauricio Neuman, Gertrudis Chale. Una pintora en el mundo. Años 1934-1954, BA, American Art Corp./ Arte al Día Ediciones, 2009, pp. 43-53, ici p. 47.

39. La revue aurait eu pour but de connecter entre eux les pays américains et de représenter « sus valores y aspectos humanos, intelectuales, artísticos, topográficos y sociopolíticos ».

40. Citée par J. Gilland, « Gertrudis Chale... », art. cit., p. 49.

41. Romualdo Brughetti, Nueva historia de la pintura y la escultura en la Argentina. De los orígenes a nuestros días, BA, Ediciones de Arte Gaglianone, 1991, p. 111.

42. "Gertrudis Chale, en sus propias palabras », in M. Neuman, Gertrudis Chale..., op. cit., pp. 10-11, document reproduit p. 10, «Contenido de la pintura » (“Apuntes de viaje”, ms., 1945).

43. Ibid.

44. Ibid.

45. Ibid.

46. Voir Ibid., document reproduit p. 11, « El indio », et « Carta a Libero Badii », Quito, 3 avril 1946 (doc. ICAA, $n^{\circ} 759659$ ), qui fit aussi le « voyage des Andes».

47. « Gertrudis Chale, en sus propias palabras », art. cit., doc. cité p. 10.

48. Témoignage de G. Chale, cité par Luis Felipe Noé, « Artes plásticas », El Mundo (BA), 20 octobre 1956 (doc. ICAA, $\mathrm{n}^{\circ}$ 739941).

49. Citée par J. Gilland, « Gertrudis Chale... », art. cit., p. 50.

50. « Gertrudis Chale, en sus propias palabras », art. cit., doc. cité p. 10. 
51. On écrira désormais AAC pour Asociación de Arte Constructivo, et TTG pour Taller TorresGarcía.

52. Dès 1932, le sculpteur Francisco Matto avait fait quant à lui, par mer, le tour de la pointe méridionale de l'Amérique et avait visité la Terre de Feu et le sud du Chili. Collectionneur de pièces d'art précolombien (cf. Arte precolombino : colección Matto, Montevideo [désormais Mtv], Museo de Arte Precolombino, 1964), c'est en 1939 que sa route croisa celle de Torres García, rencontre décisive pour toute la suite de sa carrière.

53. Voir Joaquín Torres García, Metafísica de la prehistoria indoamericana, Mtv, Publicaciones de la AAC, Talleres Gráficos "Sur", 1939, où il mobilise la littérature archéologique et anthropologique à sa disposition.

54. Joaquín Torres García, «La Escuela del Sur» (févr. 1935), in Universalismo constructivo. Contribución a la unificación del arte y de la cultura de América, [BA, Editorial Poseidón, 1944] Madrid, Alianza Editorial, 1984, vol. 1, leçon 30, pp. 193-198, avec le dessin de la carte inversée de l'Amérique du Sud, qui date de 1936.

55. À propos des phases 1941-1943, 1945-1947 et 1949, où la représentation figurée est travaillée par Torres García, Juan Fló ("Joaquín Torres García y el arte prehispánico », in Imaginarios Prehispánicos en el Arte Uruguayo : 1870-1970, Mtv, Fundación MAPI, 2006, cat. exp., pp. 33-75) parle, p. 70, de "peinture construite"; sans doute vaudrait-il mieux la qualifier de "constructive», selon les titres des tableaux eux-mêmes: Puerto, Flores, Figuras, Barco constructivos. Pour les paysages des années 1945-1947, du port et de la ville de Montevideo, le qualificatif metafísico des toiles est peut-être source de malentendu; il n'est pas sûr que son auteur fasse référence à la "peinture métaphysique » d'un Giorgio de Chirico, si l'atmosphère des œuvres y font penser.

56. Joaquín Torres García, La tradición del hombre abstracta (doctrina constructivista), ms. calligraphié et dessiné en fac-similé, Mtv, AAC, 1938 (Mtv, Ediciones 'La Regla de Oro', 1977, édition en fac-similé), avec plusieurs dessins relevant de la phase indo-américaine de Torres García, parmi d'autres, « universels ».

57. Contre les interprétations de Margit Rowell («Order and Symbol. The European in American Sources of Torres-García's Constructivism ", in Torres-García : Grid-Pattern-Sign. Paris-Montevideo, 1924-1944, Londres, Arts Council of Great Britain, 1985, pp. 9-20 [cat. exp. Hayward Gallery]), reprises par Barbara Braun ("Joaquín Torres-García: The alchemical grid », in Id., Pre-columbian Art and the Post-Columbian World: Ancient American Sources of Modern Art, New York, Harry N. Abrams, 1993, pp. 251-291), Jacqueline Barnitz (« Torres-Garcia’s Constructive Universalism and the Abstract Legacy », in Id., Twentieth-Century Art of Latin America, Austin, University of Texas Press, 2001, pp. 127-140) et Mari Carmen Ramirez («Inversions : The School of the South», in Id. et Héctor Olea, Inverted Utopias: Avant-Garde Art in Latin America, New Haven-Houston, Yale University Press/The Museum of Fine Arts, 2004, cat. exp., pp. 73-83), qui, au prix d'une torsion de la pensée torrésienne, font de son œuvre la conjonction d'une double découverte à Paris (du néoplasticisme et des arts préhispaniques), l'expression d'une modernité depuis une périphérie ou l'émergence d'une avant-garde latino-américaine, voire latino-américaniste, nous sommes plus proche de l'analyse de Juan Fló : « Joaquín Torres García y el arte prehispánico », art. cit., et Id., Torres García en (y desde) Montevideo, Mtv, Arca, 1991, qui s'attache à dégager la complexité de la pensée de Torres García, au plus près de ses nombreux écrits, et de Mario Gradowczyk, TorresGarcía, utopía y transgresión, Mtv, Museo Torres García, 2007, dont la monographie qu'il consacre à l'artiste suit la chronologie de l'œuvre peinte, telle qu'on peut l'établir en l'absence de catalogue raisonné.

58. Voir Joaquín Torres García, «Arte precolombiano ", Círculo y Cuadrado (segunda época). Revista de la Asociación de Arte Constructiva, 1, mai 1936, pp. 4-5, où il commente l'exposition de la collection d'art précolombien de Rafael Fosalba, présentée à l'Ateneo de Montevideo.

59. Joaquín Torres García, «El arte de América » (août 1940), in Universalismo constructivo..., op. cit., vol. 2, leçon 127, pp. 697-702 ; Id., «El nuevo arte de América (inédito)» (avril 1942), Apex (Mtv), 
1, juill. 1942, pp. 11-16 (repris dans Universalismo constructivo..., op. cit., vol. 2, leçon 148, datée déc. 1942, pp. 816-822) ; Id., «La Regla abstracta » (ms. calligraphié et dessiné en fac-similé, 5 févr. 1946, 12 p.), in Nueva escuela de Arte del Uruguay. Pintura y arte constructivo, contribución al arte de las tres Américas. - The New Art School of Uruguay. Painting and constructive art. Contribution to the art of the three Americas[.] - Nouvelle école d'art de l'Uruguay. Peinture et art constructif[.] Contribution à l'art des trois Amériques, Mtv, Publicaciones de la AAC, Talleres LIGU, 1946.

60. Voir les dessins suivants: Teogonía indoamericana (1937); Pachacamac Dios incaico (1938), Indoamérica/Pachamama (1938), qui illustrent le $\mathrm{n}^{\circ}$ 7, sept. 1938, de Círculo y Cuadrado, pp. 2-3 ; Arte constructivo (1938), Indoamérica (1938), Pachamama (1938) ; Indoamérica (huile sur cuir, 1941) ; et les bois peints: Inti, Pachamama et Idea ( ca 1944), le dernier ne relevant pas de l'iconographie torrésienne indo-américaine, ce qui place cette triade dans un horizon qui subsume le référent américain des deux premiers.

61. Se reporter en particulier à Notes sobre Art, Gérone, impr. Rafael Masó, 1913 : il y signalait déjà l'art précolombien, aux côtés des primitifs italiens, de la peinture grecque et égyptienne, des mosaïques byzantines, des arts primitifs ou préhistoriques, pour indiquer son souci de renouer avec une conception sacrée de l'art et de développer une peinture non imitative de la nature.

62. J. Fló, « Joaquín Torres García y el arte prehispánico », art. cit., p. 34.

63. On peut considérer la série «Heroes, hombres y monstruos» (1939-1945), où sont portraiturés de grandes figures du monde des lettres, des arts et de la musique (Rabelais, Velázquez, Mozart, etc.) - et quelques fossoyeurs de celui-ci : Hitler, Staline, Napoléon? -, comme une tentative désespérée de conjurer l'effondrement de la culture européenne, en une sorte de catharsis.

64. Voir B. Braun, Pre-columbian Art..., op. cit., qui consacre aussi un chapitre à Frank Lloyd Whright et à Diego Rivera. On pourrait y ajouter, au moins, Josef Albers, Adolph Gottlieb et Barnett Newman.

65. Cette séquence chronologique indique : 1935, la date finale de la conception du projet ; 1936, celle de l'œuvre réalisée ; 1937, celle de son érection en tant que monument public. Voir Jacques Poloni-Simard, «Nota sobre la fecha del Monumento cósmico, de Joaquín Torres García, y sus implicaciones interpretativas ", à paraître.

66. Il est à noter que les travaux des élèves de Torres García, publiés dans les quatre, voire cinq premiers numéros de Círculo y Cuadrado (mai 1936 - sept. 1937), ne comportent pas de référence à l'art préhispanique; il faut attendre le $n^{\circ} 7$ (sept. 1938), peut-être le $n^{\circ} 6$ (mars 1938), pour que le moment indo-américain soit illustré par eux également ; ils s'en éloigneront ultérieurement.

67. Cf. La decoración mural del pabellón Martirené de la colonia Saint Bois. Pinturas murales del pabellón hospital J. J. Martirené de la colonia Saint Bois, Mtv, Talleres Gráficos Sur, 1944 (la « brève notice » de présentation signale 19 artistes et 27 murales). Voir aussi : Murales TTG, Mtv, Museo Gurvich, 2007 (cat. exp.), et María Laura Bulanti, El Taller Torres-García y los murales del Hospital Saint-Bois. Testimonios para su historia, Mtv, Librería Linardi y Risso, 2008, qui inventorie 21 artistes et 35 murales. Dans une lettre à Sergio de Castro du 8 septembre [1944], Torres en signalait 36 (Archives Sergio de Castro). Je remercie Mme Dominique de Castro de m'avoir donné accès à cette correspondance.

68. El sol, El pez, Locomotora blanca, Pacha Mama, Pax in lucem, Forma. Après leur déposition en 1973 en vue de leur préservation et restauration, les murales de Torres García furent détruites en 1978 dans l'incendie du musée d'Art moderne de Rio de Janeiro, où elles avaient été exposées avec d'autres œuvres de l'artiste, au retour de l'exposition qui avait eu lieu au musée d'art moderne de la Ville de Paris en 1975 (voir Torres-García. Obras destruidas en el incendio del Museo de Arte Moderno de Rio de Janeiro, Mtv, Fundación Torres-García, s. d. [1981]).

69. L'anonymat que revendiquait Joaquín Torres García ne renvoie pas tant à l'absence de signature (lui-même signait ses œuvres - tout comme, le plus souvent, ses élèves -, auxquelles il ajoutait fréquemment la mention $\mathrm{AAC}$, même après la dissolution de l'association d'art 
constructif, utilisant les lettres d'un alphabet qu'il avait inventé) qu'à ce caractère des œuvres laissées par les artistes des sociétés primitives, préhistoriques et, dans l'immense majorité des cas, des périodes anciennes, en tant qu'expression des croyances du groupe.

70. Catalunya eterna (1913), L'Edat d'Or de la humanitat (1915), Les artes (1916), Lo temporal no es mes que simbol (1916). La cinquième, La indústria (1917), qui ne fut pas réalisée, introduisait la mythologie moderne (urbaine, industrielle, technique, ouvrière); elle provoqua la rupture du contrat qui liait Torres et la Généralité de Catalogne.

71. La structure de chaque mural est définie par les aplats de couleurs différentes (délimités par un trait noir) plutôt que par une trame de lignes orthogonales comme dans les constructivos " canoniques ", et les objets représentés en rapport avec le thème urbain, imposé par le maître, sont eux-mêmes fragmentés et obéissent au principe torrésien de leur schématisation, plutôt qu'ils ne sont à proprement parler des symboles.

72. Ainsi qualifiait-il à cette date la peinture murale, par opposition à la peinture de chevalet.

73. Joaquín Torres García, «Significado del Monumento Cósmico del Parque Rodó y de los murales de Saint Bois ", Marcha (Mtv), 250, 15 septembre 1944.

74. Cf. 500a conferencia de las dadas por J. Torres García en Montevideo entre los años 1934 y 1940, Mtv, A.A.C., Impresora L.I.G.U., 1940 (conférence prononcée le 12 novembre 1939); dès la fin de l'année 1938, Torres García manifestait son découragement: voir Manifiesto 2 constructivo 100\%, Mtv, Publicaciones de la AAC, 1938 (texte daté du mois de décembre).

75. C'est le TTG plutôt que la AAC, qui peut être comparé au Bauhaus ou au Black Mountain College. Celle-ci est en effet, aux yeux de son promoteur, autant sinon plus une sorte d'école philosophique grecque, un "centre de culture artistique», un "institut» rassemblant les adeptes d'une doctrine (constructiviste) ou un cénacle regroupant des disciples autour de leur maître qu'un simple mouvement artistique et un atelier d'artiste réunissant des élèves autour de leur professeur bousculant les codes de la pratique artistique et les frontières entre beaux-arts et arts décoratifs et appliqués.

76. Matto, el misterio de la forma, Mtv, Galería Óscar Prato, 2007 (cat. exp.) ; Gabriel Pérez-Barreiro (éd.), Francisco Matto : The Modern and the Mythic, Austin, Blanton Museum of Art, 2009 (cat. exp.).

77. César Paternosto, Abstraction. The Amerindian Paradigm, Bruxelles, Société des Expositions du Palais des Beaux-Arts de Bruxelles/IVAM - Institut Valencià d'Art Modern, 2001 (cat. exp.), avec une contribution de Cecilia de Torres, "Pilgrimaje of the Sources of Amerindian Art», pp. 247-264.

\section{RÉSUMÉS}

Le « voyage d'Europe » comme étape initiatique dans la formation des artistes latino-américains à la fin du XIXe et pendant la première moitié du XXe siècle est bien connu. Or, à partir des années 1920, une autre destination apparaît : le Nord-Ouest argentin et les pays andins voisins. Certes secondaire, cet intérêt pour le monde andin accompagne les débats autour de l'art national, traduit la quête de «lo propio » et révèle les interrogations sur l'identité de l'art argentin, uruguayen et au-delà latino-américain. Inscrivant à l'agenda des artistes d'autres thèmes et réalités, le "voyage des Andes" vient enrichir le panorama de ces deux scènes artistiques et déplace l'analyse habituellement centrée sur les binômes nationalisme vs cosmopolitisme, académie vs avant-garde. Seront envisagés Luis Perlotti (1890-1969), sculpteur 
indigéniste, qui illustre la théorie « Eurindia » de Ricardo Rojas ; Antonio Berni (1905-1981) qui, à partir d'une interrogation sur la thématique, développe une perspective sociale et critique; Gertrudis Chale (1898-1954), peintre autrichienne installée en Argentine, dont la vision du monde andin est plus anthropologique; et, enfin, quelques disciples de l'Uruguayen Joaquín Torres García (1874-1949), le père de l'art constructif universel, dans le cadre de son projet «IndoAmérica ».

El "viaje a Europa", en tanto etapa initiática en la formación de los artistas latinoamericanos es bien conocido. Ahora bien, a partir de los años 1920, aparece otro destino : el Noroeste argentino y los países andinos vecinos. Ciertamente secundario, este interés por el mundo andino acompaña los debates entorno al arte nacional, traduce la búsqueda de lo propio y revela las interrogaciones sobre la identidad del arte argentino, uruguayo y latinoamericano. Ubicando en el agenda de los artistas otros temas y otras realidades, el "viaje a los Andes" enriquece el panorama de estas dos escenas artísticas y desplaza el análisis habitualmente centrado en las tensiones entre nacionalismo vs cosmopolitismo, academia vs. vanguardia. Presentaremos Luis Perlotti (1890-1969), escultor indigenista,ilustrando la teoria "Eurindia" de Ricardo Rojas; (Antonio Berni(1905-1981), quien desarrolla una perspectiva social crítica a partir de una interrogación sobre la temática por representar; Gertrudis Chale (1898-1954), pintora austríacaradicada en la Argentina, cuya visión del mundo andino es más bien antropológica ; y, en fin, algunos discípulos del Uruguayo Joaquín Torres García (1874-1949), padre del Arte constructivo universal, que con él han participado a su proyecto indoamericano.

\section{INDEX}

Mots-clés : Luis Perlotti, Antonio Berni, Gertrudis Chale, Joaquín Torres García, Ricardo Rojas, art national, indigénisme, art latino-américan, relations Río de la Plata-Andes

Palabras claves : Luis Perlotti, Antonio Berni, Gertrudis Chale, Joaquín Torres García, Ricardo Rojas, arte nacional, indigenismo, arte latinoamericano, relaciones Río de la Plata-Andes

\section{AUTEUR}

JACQUES POLONI-SIMARD

EHESS-CERMA/Mondes américains 